\title{
PHASE PORTRAITS OF SIR EPIDEMIC MODELS WITH VERTICAL TRANSMISSIONS AND LINEAR TREATMENT RATES
}

by

\author{
Marvin Hoti, BSc, Ryerson University, 2015 \\ A thesis presented to Ryerson University \\ in partial fulfilment of the \\ requirements for the degree of \\ Master of Science \\ in the program of \\ Applied Mathematics
}

Toronto, Ontario, Canada, 2017

(C)Marvin Hoti, 2017 


\section{Author's Declaration}

I hereby declare that I am the sole author of this thesis. This is a true copy of the thesis, including any required final revisions, as accepted by my examiners. I authorize Ryerson University to lend this thesis to other institutions or individuals for the purpose of scholarly research.

I further authorize Ryerson University to reproduce this thesis by photocopying or by other means, in total or in part, at the request of the other institutions or individuals for the purpose of scholarly research.

I understand that my thesis may be made electronically available to the public. 


\title{
PHASE PORTRAITS OF SIR EPIDEMIC MODELS WITH VERTICAL TRANSMISSIONS AND LINEAR TREATMENT RATES
}

\author{
Marvin Hoti \\ Master of Science, 2017 \\ Applied Mathematics Program \\ Ryerson University
}

\begin{abstract}
Susceptible-infective-removed epidemic models with horizontal and vertical transmissions and linear treatment rates are investigated. All the ranges of the parameters involved in the models for the infection-free equilibrium and the epidemic equilibrium to be positive are found. Like the previous results on the models without vertical transmissions or the linear treatments, we study stability of these equilibria. The novelty is that we justify that these positive equilibria are stable focuses or stable nodes under suitable conditions on the parameters. These results provide more detailed descriptions of behaviours of the epidemic diseases near the equilibria. Our results will exhibit the effect of the vertical transmissions and the linear treatment rates on the epidemic models. Some simulations results are provided to understand the phase portraits near the equilibria.
\end{abstract}




\section{ACKNOWLEDGEMENTS}

Firstly, I would like to express my gratitude to my supervisor Dr. Kunquan Lan. He has given me great support over the years from my BSc degree all the way to my MSc degree. Thank you, for giving me your time and always being willing to help me with a problem. One of the most valuable things I have learned from you is the ability to do research, and the significance of organizing and presenting the work in a clear manner. This has led me to become more "mathematically mature". Thank you for creating a good learning environment for me and your students. It is clear to see that you are passionate about your research and that you want to help others be successful as well. Because of this I feel more confident about pursing my $\mathrm{PhD}$ studies in mathematics, and a lot of that motivation to pursue my $\mathrm{PhD}$ stems from working with you. I would also like to thank Dr. Xi Huo for helping me with my research and providing feedback on my work, and helping me to understand some of the concepts that I had to use for my thesis.

I want to thank the faculty of Mathematics at Ryerson University for giving me an opportunity to study here and for providing me with financial assistance. Also, I would like to thank the professors that I had the pleasure to be taught by. I would also like to thank Kathy Peter, and Teresa Lee for always being able to help me with anything.

Lastly, I would like to thank the members of the examining committee, Dr. Kunquan Lan, Dr. Silvana Ilie, Dr. Pawel Pralat, and Dr. Dzung Minh Ha for their time and commitments to review my thesis. 


\section{Preface}

This thesis is a record of part of the research carried out by the author during the academic years of 2015-2017. It is submitted according to the regulations for the degree of Master of Science at Ryerson University.

Almost all of the results of this thesis are the original work of the author with the exception of several results specifically mentioned in the text and attributed there to the authors concerned.

Chapters 1 and 2 contain the preliminary materials, while chapters 3,4 and 5 consist of new results. 


\section{Contents}

Declaration $\quad$ ii

Abstract

Acknowledgements iv

Preface v v

1 Introduction 1

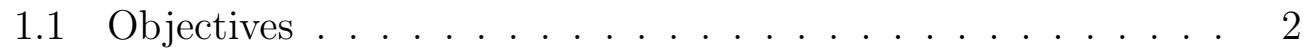

1.2 Methodology . . . . . . . . . . . . . . . . 3

2 Theory of dynamical systems 5

2.1 Introduction . . . . . . . . . . . . . . 5

2.2 Definition of autonomous differential equation . . . . . . . 7

2.3 Equilibria of differential equations . . . . . . . . . . . 7

2.4 Phase plane analysis . . . . . . . . . . . . . . . . . . 8

3 Introduction to the epidemic models $\quad 13$ 
3.1 Background of the epidemic model . . . . . . . . . . . 13

3.2 Construction of the model . . . . . . . . . . . . . . 15

3.3 Previous results . . . . . . . . . . . . . . . . . . . 17

3.4 Positive equilibria of the model f . . . . . . . . . . . . . . . 18

4 Qualitative behaviour of the equilibrium points 23

4.1 Phase portraits of the equilibria points . . . . . . . . . 23

$\begin{array}{lll}5 & \text { Discussion } & 41\end{array}$

$\begin{array}{ll}\text { Bibliography } & 42\end{array}$ 


\section{List of Figures}

3.1 Flow diagram of the SIR model. . . . . . . . . . . . . 16

4.1 Phase portrait for $4.1 .1(2) \ldots \ldots . \ldots . . \ldots 27$

4.2 The unique solution of $h(x)=0 \ldots \ldots$. . . . . . . . . 28

4.3 Phase portrait of theorem 4.1.6 $\left(\mathrm{H}_{2}\right)(\mathrm{ii})$ and $2(\mathrm{iii})$ respectively. 36

4.4 Phase portrait of theorem 4.1.9. . . . . . . . . . . . . . 40 


\section{Chapter 1}

\section{Introduction}

Infectious-diseases have been a concern for a while now. When they enter a population it can be very difficult to control the spread. A well known example of this is the bubonic plague that affected most of Europe in the mid 14th century. This disease was deadly and it spread quickly, it wiped out more than half of the population in Europe in just a few years. A more recent epidemic that occurred in the early 2000. Was the SARS epidemic, or severe acute repository syndrome. This was first identified in Asia and spread quickly around the world affecting over 5000 people. Epidemic models help us understand the interaction of diseases when they affect a population. Through mathematical analysis, we can understand how treatment can help eradicate or maintain the spread of disease. Treatments (public health intervention) such as isolation or quarantine are useful methods to prevent epidemic diseases. An example of this can be the Ebola out-break that occurred recently, which originated in Africa. All the individuals that were 
infected at the time were quickly quarantined even though no cure was readily available or existed at the time. The purpose was to prevent it from spreading, and eradicate the disease that way.

\section{$1.1 \quad$ Objectives}

Recently there have been a few epidemic models which have been introduced, each has their advantages and disadvantages and some are more suitable to represent a specific class of diseases over others. The main objective of this thesis are the following

- 1. To modify a previously introduced SIR epidemic model and study its dynamical behaviour.

- 2. To find all of the positive equilibria of the model and study the qualitative behaviour of each equilibria through each of the parameters involved.

The model that we modify is the SIR-epidemic model introduced in [12]. We add the following treatment function $T(I)=h I$, where $0 \leq h<\infty$. We

define $h$ later in the paper. It is important to note that we can not combine $h$ with our other parameters. 


\subsection{Methodology}

To perform our mathematical analysis we will use the theory and methods introduced in chapter 2 . We will reduce our system of three non-linear differential equations to just two non-linear differential equations, letting us analyse the model in $\mathbb{R}^{2}$. We will introduce the reproduction number $\mathcal{R}_{0}$ and through it we will give the ranges of parameters for the model to have positive equilibria. After we have determined the equilibria we will study the stability of the model for each of its equilibrium.

\section{Thesis organization}

- Chapter 1: We have provided a brief introduction to the motivation and study of epidemic models, our objective and method.

- Chapter 2: We introduce the mathematical theory, definitions and methods that we will use through out our paper to determine the equilibria of the model and the theory required to study the stability of each equilibrium.

- Chapter 3: We give some background on epidemic models and motivation for them. We introduce one of the very first epidemic models. We show a flow diagram to illustrate how we build an SIR epidemic model. The parameters and their biological meanings are introduced. We introduce the model that we study, and some previous results are mentioned. Lastly, we give all the positive equilibria of the model and the conditions for the parameters involved. 
- Chapter 4: We study the stability for each positive equilibria. Some figures are shown to illustrate our results.

- Chapter 5: This chapter contains the discussion of our results. 


\section{Chapter 2}

\section{Theory of dynamical systems}

\section{$2.1 \quad$ Introduction}

In this chapter we will introduce the mathematical theory that we will use throughout our thesis. In particular, the theory of dynamical systems will allow us to analyse our model. We introduced the following theory because the model we study is a non-trivial dynamical system composed of ordinary non-linear differential equations. Thus it is not possible to find explicit solutions of the system in question. The theory we introduce will allow us to study the behaviour of the system near its equilibria. This will allow us to better understand the long term behaviour of our model. 
Consider the following planar system

$$
\left\{\begin{array}{l}
\dot{x}(t)=f(x(t), y(t)), \\
\dot{y}(t)=g(x(t), y(t)),
\end{array}\right.
$$

subject to the initial value condition:

$$
(x(0), y(0))=\left(x_{0}, y_{0}\right)
$$

where $f, g \in C^{1}\left(\mathbb{R}^{2}\right)$.

Definition 2.1.1. $(x, y)$ is said to be a solution of (2.1.1)-(2.1.2) if $x, y \in$ $C^{1}\left(\mathbb{R}_{+}\right)$and satisfy (2.1.1)-(2.1.2). A solution $(x, y)$ is said to be positive if $x, y \in P$, where

$$
P=\left\{x \in C^{1}\left(\mathbb{R}_{+}\right): x(t) \geq 0 \quad \text { for } t \in \mathbb{R}_{+}\right\}
$$

Since $f, g \in C^{1}\left(\mathbb{R}^{2}\right)$, it is well known that for each initial value $\left(x_{0}, y_{0}\right) \in \mathbb{R}^{2}$, (2.1.1)-(2.1.2) has a unique solution. Moreover, if $f$ and $g$ satisfy

$$
f(0, y) \geq 0 \text { and } g(x, 0) \geq 0 \text { for } x, y \in \mathbb{R}_{+},
$$

then for each initial value $\left(x_{0}, y_{0}\right) \in \mathbb{R}_{+}^{2}$, the unique solution of (2.1.1)-(2.1.2) is positive (see [15, Proposition B.7]).

Let us introduce the following autonomous dynamical system

$$
\left\{\begin{array}{l}
\dot{x}=f(x, y), \\
\dot{y}=g(x, y),
\end{array}\right.
$$




\subsection{Definition of autonomous differential equa- tion}

An autonomous system is a system of ordinary differential equations of the form, $\dot{x}(t)=f(x(t))$. That is, it is a system of ordinary differential equations which do not explicitly depend on the independent variables. Where $t$ represents time.

\subsection{Equilibria of differential equations}

An equilibrium point of a system of differential equations is a constant solution of the system. They allow us to understand the behaviour of the system in a neighbourhood of each of the equilibrium points. With respect to epidemic models, they are of interest since they represent resting or stationary states of the system. For example, if there exists an equilibrium point of the form $(x, 0)$, where $x>0$, then this would represent a disease-free equilibrium point. This would represent the state when the disease dies out.

Definition 2.3.1. A point $(x, y) \in \mathbb{R}_{+}^{2}$ is said to be an equilibrium point of (2.1.3) if $(x, y)$ satisfies $f(x, y)=0$ and $g(x, y)=0$. It is said to be a positive equilibrium point if $x \geq 0$ and $y \geq 0$; an interior equilibrium point if $x>0$ and $y>0$. 


\subsection{Phase plane analysis}

In order to analyse the behaviour of our system near each of the equilibrium points we need to introduce the following well-known qualitative theory. Before we introduce some of the results we will use, let us first introduce the linearization of a non-linear system about its equilibrium point.

Definition 2.4.1. [20] Linearization is a method that will allow us to analyse the local stability of an equilibrium point of a system of non-linear differential equations.

Consider system (2.1.3), a first order autonomous differential system with two variables $x$ and $y$. The local stability of an equilibrium point is determined by the eigenvalues of the Jacobian matrix. The functions $f$ and $g$ are expanded using Taylor formula about the equilibrium point $(\bar{x}, \bar{y})$. Assume that $f$ and $g$ have continuous-second order partial derivatives in an open set containing the point $(\bar{x}, \bar{y})$. Let $u=x-\bar{x}$, and $v=y-\bar{y}$. Then

$$
\begin{aligned}
& \frac{d u}{d t}=f(\bar{x}, \bar{y})+f_{x}(\bar{x}, \bar{y}) u+f_{y}(\bar{x}, \bar{y}) v+f_{x x}(\bar{x}, \bar{y}) \frac{u^{2}}{2}+\ldots \\
& \frac{d v}{d t}=g(\bar{x}, \bar{y})+g_{x}(\bar{x}, \bar{y}) u+g_{y}(\bar{x}, \bar{y}) v+g_{x x}(\bar{x}, \bar{y}) \frac{u^{2}}{2}+\ldots
\end{aligned}
$$

where

$f_{x}(\bar{x}, \bar{y})=\left.\frac{\partial f(x, y)}{\partial x}\right|_{x=\bar{x}, y=\bar{y}}$ and $g_{x}(\bar{x}, \bar{y})=\left.\frac{\partial g(x, y)}{\partial x}\right|_{x=\bar{x}, y=\bar{y}}$ and so on. Recall from definition 2.3.1, that an equilibrium point $(\bar{x}, \bar{y})$ of the system (2.1.3) satisfies $f(\bar{x}, \bar{y})=0$ and $g(\bar{x}, \bar{y})=0$. 
The system linearized about the equilibrium $(\bar{x}, \bar{y})$ is

$$
\frac{d Z}{d t}=A Z
$$

where $Z=(u, v)^{T}$ and $A$ is the Jacobian matrix evaluated at the equilibrium point. see (2.4.1) below.

Now we introduce some results on stability and phase portraits of planar systems near the equilibria in qualitative theory see $[1,13,15]$.

We denote by $A(x, y)$ the Jacobian matrix of $f$ and $g$ at $(x, y)$.

$$
A(x, y)=\left(\begin{array}{ll}
\frac{\partial f}{\partial x} & \frac{\partial f}{\partial y} \\
\frac{\partial g}{\partial x} & \frac{\partial g}{\partial y}
\end{array}\right)
$$

and by $|A(x, y)|$ and $\operatorname{tr}(A(x, y))$ the determinant and the trace of $A(x, y)$ respectively.

The characteristic polynomial of $A(x, y)$ is

$$
\lambda^{2}-\operatorname{tr}(A(x, y))+|A(x, y)|
$$

It is well known that the solutions of a planar system near its equilibria $(\bar{x}, \bar{y})$ can be studied by the eigenvalues of $A(x, y)$, which are determined by $|A(x, y)|$ and $\operatorname{tr}(A(x, y))$.

Definition 2.4.2. An equilibrium point $\left(x^{*}, y^{*}\right)$ is said to be globally asymptotically stable if it is locally asymptotically stable and each solution $(x, y)$ of (2.1.1)-(2.1.2) with $\left(x_{0}, y_{0}\right) \in \mathbb{R}_{+}^{2}$ converges to $\left(x^{*}, y^{*}\right)$ in $\mathbb{R}^{2}$, that is, $\lim _{t \rightarrow \infty}(x(t), y(t))=\left(x^{*}, y^{*}\right)$. 
Definition 2.4.3. An equilibrium point is a node if the Jacobian matrix has two distinct real eigenvalues with the same sign. If the eigenvalues are real and negative then it is a stable node. The solution will approach the equilibrium point regardless of the starting point.

Definition 2.4.4. An equilibrium point is a focus if the Jacobian matrix has complex eigenvalues with non-zero real parts. The solutions start from any point other then the equilibrium point and spiral towards the equilibrium point.

Definition 2.4.5. An equilibrium point is a saddle node if the Jacobian matrix has one zero eigenvalue. Two equilibrium points, a saddle and a node collide with each other and disappear.

The following results can be found in [13] and have been used in $[4,8,10$, $11,19]$.

Lemma 2.4.1. [20] If $\left(x^{*}, y^{*}\right)$ is an equilibrium of (2.1.1), then the following assertions hold.

(i) If $\left|A\left(x^{*}, y^{*}\right)\right|<0$, then $\left(x^{*}, y^{*}\right)$ is a saddle of (2.1.1).

(ii) If $\left|A\left(x^{*}, y^{*}\right)\right|>0, \operatorname{tr}\left(A\left(x^{*}, y^{*}\right)\right)<0$ and $\left(\operatorname{tr}\left(A\left(x^{*}, y^{*}\right)\right)\right)^{2}-4\left|A\left(x^{*}, y^{*}\right)\right| \geq 0$ then $\left(x^{*}, y^{*}\right)$ is a stable node of (2.1.1).

(iii) If $\left|A\left(x^{*}, y^{*}\right)\right|>0, \operatorname{tr}\left(A\left(x^{*}, y^{*}\right)\right)<0$ and $\left(\operatorname{tr}\left(A\left(x^{*}, y^{*}\right)\right)\right)^{2}-4\left|A\left(x^{*}, y^{*}\right)\right|<0$ then $\left(x^{*}, y^{*}\right)$ is a stable focus of (2.1.1). (iv) If $\left|A\left(x^{*}, y^{*}\right)\right|>0$ and $\operatorname{tr}\left(A\left(x^{*}, y^{*}\right)\right)<0$, then $\left(x^{*}, y^{*}\right)$ is locally asymptotically stable. 
Lemma 2.4.2. Assume that each positive solution of (2.1.1)-(2.1.2) with $\left(x_{0}, y_{0}\right) \in \mathbb{R}_{+}^{2}$ is contained in a bounded closed subset $B$ of $\mathbb{R}^{2}$. Assume that $B$ contains only one equilibrium $\left(x^{*}, y^{*}\right)$ of $(2.1 .1)$ and $\left(x^{*}, y^{*}\right)$ belongs to the boundary of $B$. Then each positive solution of (2.1.1)-(2.1.2) converges to $\left(x^{*}, y^{*}\right)$.

Definition 2.4.6. A map $T: \mathbb{R}^{2} \rightarrow \mathbb{R}^{2}$ defined by $T(x, y)=(f(x, y), g(x, y))$ is said to be regular if $T$ is one to one and onto, $T$ and $T^{-1}$ are continuous and $|A(x, y)| \neq 0$ on $\mathbb{R}^{2}$. If $T$ is regular, then the following transformation

$$
\left\{\begin{array}{c}
u=f(x, y), \\
v=g(x, y)
\end{array}\right.
$$

is said to be a regular transformation. If (2.1.1) is changed into another system under suitable regular transformations, then the two systems are said to be equivalent. It is known that under regular transformations, the topological structures of solutions of a planar system near equilibria including a variety of dynamics like saddles, topological saddles, nodes, saddle-nodes, foci, centers, or cusps remain unchanged.

Lemma 2.4.3. [8] Let $\left(x^{*}, y^{*}\right)$ be an equilibrium of (2.1.1). Assume that $\left|A\left(x^{*}, y^{*}\right)\right|=0, \operatorname{tr}\left(A\left(x^{*}, y^{*}\right)\right) \neq 0$ and (2.1.1) is equivalent to the following system

$$
\left\{\begin{array}{l}
\dot{u}=p(u, v), \\
\dot{v}=\varrho v+q(u, v)
\end{array}\right.
$$


with an isolated equilibrium point $(0,0)$, where $\varrho \neq 0$, $p(u, v)=\sum_{i+j=2, i, j \geq 0}^{\infty} a_{i j} u^{i} v^{j}$ and $q(u, v)=\sum_{i+j=2, i, j \geq 0}^{\infty} b_{i j} u^{i} v^{j}$ are convergent power series. If $a_{20} \neq 0$, then $\left(x^{*}, y^{*}\right)$ is a saddle-node of (2.1.1). 


\section{Chapter 3}

\section{Introduction to the epidemic models}

In the first section of this chapter we will introduce the background of the epidemic model that we will study. We will show how the model is constructed and the motivation behind it. In the second part of the section we will present our model of interest. The model is an extension of a model studied in [12].

\subsection{Background of the epidemic model}

The study of infectious disease models or epidemic models is useful in understanding the spread of disease in a population, or the interaction of disease in a community. The following paper [5] introduces this idea using basic epidemic models. Kermack introduced one of the first SIR model composed 
of non-linear ordinary differential equations to explain the interaction that diseases such as the great plague (1665-1666), the cholera epidemic in London (1865), and the plague in Bombay (1906) had on a population. The following is the model Kermack introduced

$$
\left\{\begin{array}{l}
\frac{d S}{d t}=-\frac{\beta S I}{N}, \\
\frac{d I}{d t}=\frac{\beta S I}{N}-r I, \\
\frac{d R}{d t}=r I
\end{array}\right.
$$

where the population is divided into three subgroups, $S, I$, and $R$. $S$ represents the portion of the population that is susceptible to the disease. $I$ represents the portion of the population that is infected or infectious. $R$ represents the portion of the population that is removed from the susceptible and infectious subgroups. Note that in this model it is assumed that once an individual has recovered from the disease (infection) they can not contract it again. They are immune to it (lifelong). It is assumed that the total population is unchanged, $S+I+R=N$, where $N$ is the total population at some time $t$. The above model is limited in the sense that it does not accurately capture a real world setting. For example, it assumes no birth-rate or natural death-rate, or treatment of any kind. Moreover, this models assumes only horizontal transmission. That is, it assumes that individuals can only be infected by direct contact. Meng and Chen [12] improved the model by introducing vertical transmission. Vertical transmission is the passing of the infection from the mother to her offspring. Further, they also introduced 
a birth-rate $b$, and a natural death-rate $d$. It is assumed that $b=d$. The above modifications to the model allows it to represent more diseases such as measles, rubella and HIV-AIDS. The following section will introduce this model and show how it is constructed and our modification on it.

\subsection{Construction of the model}

In this section we introduce the model studied in [12], and how it is constructed. We show our modification to the model and its significance. The following model is the SIR model in [12].

$$
\left\{\begin{array}{l}
\dot{S}=-\beta S I-d S+p b I+b(S+R), \\
\dot{I}=\beta S I-d I-r I+q b I, \\
\dot{R}=r I-b R
\end{array}\right.
$$

where $S(t), I(t), R(t)$ represent the densities of the three subgroups of the population at time $t \geq 0$ as mentioned earlier.

- $\beta>0$ denotes the effective per capita contact rate of infective individuals (effective rate of transmission).

- $q \in[0,1]$ denotes the fraction of the infected unborn or newly born offspring of the infective parent.

- $b>0$ denotes the birth rate of the susceptible population, which is assumed to equal the death rate, to keep the total population unchanged. 
- $r>0$ denotes the recovery rate of the infective individuals.

- $p \in[0,1]$ denotes the proportion of offspring's of infective parents that are susceptible individuals.

- $d>0$ denotes the natural death rate.

The following diagram can aid the reader in understanding how the model was set up.

Figure 3.1: Flow diagram of the SIR model.

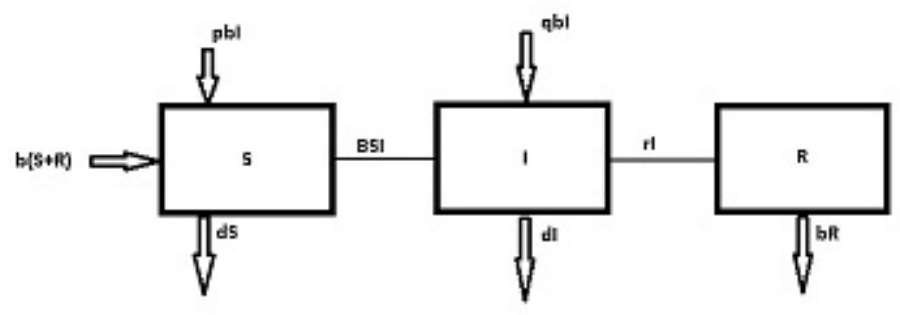

We modify the model by introducing a linear treatment rate $h I$, where $h \geq 0$ is a constant representing the capacity of the treatment for a disease in a community. It is important to note that treatment isn't necessarily done in the form of antibiotics or medication of any sort. A form of quarantine or isolation would also be considered as a form of treatment. Treatment here is assumed to be proportional to the level of the infectious population. Thus, the model becomes 


$$
\left\{\begin{array}{l}
\dot{S}=-\beta S I-d S+p b I+b(S+R), \\
\dot{I}=\beta S I-d I-r I+q b-h I, \\
\dot{R}=r I-b R+h I,
\end{array}\right.
$$

Note that the population has a constant size which we normalize to unity. So $S(t)+R(t)+I(t)=1$ for $t \geq 0$. Further, $p+q=1$, so we have $R=1-(S+R)$, and $p=1-q$. For simplification, we let $x(t)=s(t)$ and $y(t)=I(t)$. Thus, the above model can be rewritten as

$$
\left\{\begin{array}{l}
\dot{x}=b-\beta x y-b x-q b y:=f(x, y), \\
\dot{y}=\beta x y-b y-r y+q b y-h y:=g(x, y),
\end{array}\right.
$$

where $x(t)$ and $y(t)$ denote the densities of the populations of the susceptible and infective respectively at time $t \geq 0$.

\subsection{Previous results}

The above model with $h=0$ and $q \in(0,1)$ becomes the SIR model only with horizontal and vertical transmissions considered by Meng and Chen [12]. They showed that the model contains a disease free equilibrium $\left(S_{0}^{*}, 0\right)$ and a positive interior equilibrium $\left(S_{1}^{*}, I_{1}^{*}\right)$. They showed that under suitable conditions the disease-free equilibrium $\left(S_{0}^{*}, 0\right)$ is unstable or locally stable, and the positive interior equilibrium or epidemic equilibrium $\left(S_{1}^{*}, I_{1}^{*}\right)$ can be unstable or locally stable. They showed these results in terms of $\mathcal{R}_{0}$, 
where $\mathcal{R}_{0}$ is the basic reproduction number. $\mathcal{R}_{0}$ is defined to be the number or infections caused by an infected individual in a completely susceptible population.

The model (3.2.3) with constant treatment rates was studied by Luo, Zhu and Lan [11]. They studied the phase portraits near the equilibria for the epidemic models with horizontal and vertical transmissions and constant treatment rates. They provided the conditions on the parameters involved and justified that under these conditions, the equilibria are stable focuses, stable nodes, saddle-nodes or cusps with dimension 2. Bogdanov-Takens bifurcations containing saddle-node bifurcations, Hopf bifurcations and homoclinic bifurcations were studied in [11].

The SIR models (3.2.3) with $q=h=0$ is the classic model only with horizontal transmissions studied by Hethcote [5], where no vertical transmission or treatment is accounted for.

\subsection{Positive equilibria of the model}

Recall that $(x, y) \in \mathbb{R}^{2}$ is an equilibrium of (3.2.3) if $f(x, y)=0$ and $g(x, y)=$ 0 . An equilibrium $(x, y)$ of (3.2.3) is said to be positive if $x \geq 0$ and $y \geq 0$; and to be a positive interior (endemic) equilibrium if $x>0$ and $y>0$.

Notation: Let

$$
\eta:=\eta(r, b, q)=r+(1-q) b \quad \text { and } q_{1}=\frac{b+r-\beta}{b} .
$$


The following result gives necessary and sufficient conditions among the parameters, which are useful to understand the conditions used in the rest of results.

Lemma 3.4.1. (1) $\beta<\eta$ if and only if either $r \leq \beta<b+r$ and $0 \leq q<q_{1}$ or $\beta<r$ and $0 \leq q \leq 1$.

(2) $\eta<\beta$ if and only if $b+r<\beta$ and $0 \leq q \leq 1$ or $r<\beta \leq b+r$ and $q_{1}<q \leq 1$

(3) $\eta \leq \beta$ if and only if either $b+r<\beta$ and $0 \leq q \leq 1$ or $r \leq \beta \leq b+r$ and $q_{1} \leq q \leq 1$

(4) $\eta \leq \beta<\eta+b$ if and only if either $b+r<\beta<2 b+r-q b$ and $0 \leq q \leq 1$ or $r \leq \beta \leq b+r$ and $q_{1} \leq q \leq 1$.

(5) $\beta=\eta$ if and only if $r \leq \beta \leq b+r$ and $q=q_{1}$.

Proof. By definition of $\eta$ and $q_{1}$ we have,

$$
\eta-\beta=b\left(\frac{b+r-\beta}{b}-q\right)=b\left(q_{1}-q\right)
$$

and,

$$
\eta+b-\beta=b\left(\frac{2 b+r-\beta}{b}-q\right)=b\left(q_{1}+1-q\right)
$$

(1) If $r \leq \beta<b+r$ then $0<q_{1}<1$ and $q_{1}=1$ if $\beta=r$. This together with $0 \leq q<q_{1}$ and equation (3.4.1) imply the result.

If $\beta<r$ then $q_{1}>1$. This together with $0 \leq q \leq 1$ and equation (3.4.1) imply the result. 
(2) If $r+b<\beta$ then $q_{1}<0$.This together with $0 \leq q \leq 1$ and equation (3.4.1) imply the result.

If $r<\beta<b+r$ then $0 \leq q_{1}<1$. This together with $q_{1}<1 \leq 1$ and equation (3.4.1) imply the result.

(3) This result follows from result (2) with $q=q_{1}$. If $q=q_{1}$, by equation (3.4.1) we have $\eta-\beta=0$.

(4) If $b+r<\beta$ then $q_{1}<0$. Since $\beta<2 b+r-q b$ then $\eta+b-\beta=$ $2 b+r-q b-\beta>0$. So $\beta<\eta+b$. Thus $\eta \leq \beta<\eta+b$.

If $r \leq \beta \leq b+r$ then $0 \leq q_{1} \leq 1$. Further, since $q \in[0,1]$ then $1-q \geq 0$. The result follows from equation (3.4.2).

(5) $r \leq \beta \leq b+r$ then $0 \leq q_{1} \leq 1$, since $q=q_{1}$ by equation (3.4.1) we have $\eta-\beta=0$. The result holds.

We denote by $\mathcal{R}_{0}$ the basic reproduction number of the model (3.2.3). Then

$$
\mathcal{R}_{0}=\frac{\beta}{\eta+h} .
$$

Lemma 3.4.2. (1) $\mathcal{R}_{0}>1$ if and only if $\eta<\beta$ and $0 \leq h<\beta-\eta$.

(2) $\mathcal{R}_{0}<1$ if and only if either $0<\beta<\eta$ and $h \geq 0$ or $\eta \leq \beta$ and $h>\beta-\eta$.

(3) $\mathcal{R}_{0}=1$ if and only if $\eta \leq \beta$ and $h=\beta-\eta$.

Proof. By definition of $\mathcal{R}_{0}$ we have,

$$
\mathcal{R}_{0}-1=\frac{\beta-\eta-h}{h+\eta}
$$


Note, that $h \geq 0$ and $\eta>0$ so $h+\eta \neq 0$. Thus (3.4.4) is well-defined.

(1) If $\eta<\beta$ and $0 \leq h<\beta-\eta$ by equation (3.4.4) we have $\mathcal{R}_{0}-1>0$. The result follows.

(2) If $0<\beta<\eta$ and $h \geq 0$ then $\beta-\eta<0$. By equation (3.4.4) we have $\mathcal{R}_{0}-1<0$.

If $\eta \leq \beta$ and $h>\beta-\eta$ then $\beta-\eta \geq 0$. By equation (3.4.4) we have $\mathcal{R}_{0}-1<0$. The result follows.

If $\eta \leq \beta$ then $\beta-\eta \geq 0$. Since $h=\beta-\eta$ by equation (3.4.4), $\mathcal{R}_{0}-1=0$. The result follows.

We prove the following main result on the number of equilibria of (3.2.3).

Theorem 3.4.3. (1) If $\mathcal{R}_{0} \leq 1$, then $(1,0)$ is the unique positive equilibrium of $(3.2 .3)$.

(2) If $\mathcal{R}_{0}>1$, then (3.2.3) has only two positive equilibria: $(1,0)$ and $(\bar{x}, \bar{y})$, where

$$
\bar{x}=\frac{\eta+h}{\beta} \quad \text { and } \bar{y}=\frac{b(\beta-\eta-h)}{\beta(b+r+h)} .
$$

Proof. It is clear that $(x, y)$ is an equilibrium of $(3.2 .3)$ if and only if $(x, y)$ satisfies the following system

$$
\left\{\begin{array}{l}
b-\beta x y-b x-q b y=0, \\
\beta x y-b y-r y+q b y-h y=0 .
\end{array}\right.
$$

For $b, r, \beta>0, q \in[0,1]$ and $h \geq 0$, it is clear that $(1,0)$ is a solution of (3.4.6) and is a positive equilibrium of (3.2.3). It is easy to see that (3.4.6) 
with $y \neq 0$ is equivalent to the following system

$$
\left\{\begin{array}{l}
b-\beta x y-b x-q b y=0, \\
\beta x-r-b-h+q b=0 .
\end{array}\right.
$$

Solving the second equation of (3.4.7) we obtain

$$
x=\frac{r+(1-q) b+h}{\beta}=\frac{\eta+h}{\beta} .
$$

This, together with the first equation of (3.4.7), implies

$$
y=\frac{b[\beta+q b-(r+b+h)]}{\beta(r+b+h)}=\frac{b(\beta-\eta-h)}{\beta(r+b+h)} .
$$

If $\mathcal{R}_{0}>1$, then by Lemma 3.4 .2 (1) we have $\eta<\beta$ and $0 \leq h<\beta-\eta$. This, together with (3.4.9), implies $y>0$. Hence, $(\bar{x}, \bar{y})$ given in (3.4.5) is a positive interior equilibrium of (3.2.3). If $\mathcal{R}_{0}<1$, then by Lemma 3.4.2 (2) we have either $0<\beta<\eta$ and $h \geq 0$ or $\eta \leq \beta$ and $h>\beta-\eta$. This implies that $\beta-\eta-h<0$ and $y<0$. Hence, (3.2.3) has no positive interior equilibria. If $\mathcal{R}_{0}=1$, then by Lemma 3.4.2 (3) we have $\eta \leq \beta$ and $h=\beta-\eta$. This, together with (3.4.9) and (3.4.8), implies $y=0$ and $x=1$. The results follow.

Theorem 3.4.3 improves [5, Theorem 6.1] and the result on the number of positive equilibria obtained in [12, section 2]. We generalize the results for positive equilibria obtained in $[12$, section 2$]$ and provide the range on the parameters involved to achieve a positive equilibrium. 


\section{Chapter 4}

\section{Qualitative behaviour of the equilibrium points}

\subsection{Phase portraits of the equilibria points}

In this section, we study the stability and phase portraits of each positive equilibrium of (3.2.3).

Let $A(x, y)$ be the Jacobian matrix of $f$ and $g$ defined in (3.2.3). By (3.2.3) and (2.4.1), we have

$$
A(x, y)=\left(\begin{array}{cc}
-\beta y-b & -\beta x-q b \\
\beta y & \beta x-\eta-h
\end{array}\right) .
$$

Note that $\eta=r+(1-q) b$, we have

$$
|A(x, y)|=\beta y(b+r+h)-b \beta x+b(\eta+h)
$$


and

$$
\operatorname{tr}(A(x, y))=-\beta(y-x)-(b+\eta+h) .
$$

We first prove the following result on the global stability and phase portraits near the infection-free equilibrium $(1,0)$ of $(3.2 .3)$.

Theorem 4.1.1. (1) If $\mathcal{R}_{0}>1$, then $(1,0)$ is a saddle of $(3.2 .3)$.

(2) If $\mathcal{R}_{0}<1$, then $(1,0)$ is a stable node of (3.2.3). Moreover, the infection-free equilibrium $(1,0)$ of $(3.2 .3)$ is globally asymptotically stable.

(3) If $\mathcal{R}_{0}=1$, then $(1,0)$ is a saddle-node of $(3.2 .3)$.

Proof. By (4.1.1) and (4.1.2) with $(x, y)=(1,0)$, we have

$$
|A(1,0)|=b(h-\beta+\eta)
$$

and

$$
\operatorname{tr}(A(1,0))=\beta-\eta-h-b
$$

(1) Since $\mathcal{R}_{0}>1$, by (4.1.3) and Lemma 3.4.2 (1), we have $|A(1,0)|<0$. The result follows from Lemma 2.4.1 (i).

(2) Since $\mathcal{R}_{0}<1$, by Lemma 3.4 .2 (2), (4.1.1) and (4.1.2) we obtain $|A(1,0)|>0$ and $\operatorname{tr}(A(1,0))<0$. Moreover, we have

$$
\begin{aligned}
\operatorname{tr}(A(1,0))^{2}-4|A(1,0)| & =(\beta-\eta-h-b)^{2}-4 b(h-\beta+\eta) \\
& =(\beta-\eta-h)^{2}-2 b(\beta-\eta-h)+b^{2}+4 b(\beta-h-\eta) \\
& =(\beta-\eta-h)^{2}+2 b(\beta-\eta-h)+b^{2} \\
& =(\beta-\eta-h+b)^{2} \geq 0 .
\end{aligned}
$$

The first result follows from Lemma 2.4.1 (ii). 
Let $B=\left\{(u, v) \in \mathbb{R}_{+}^{2}: u+v \leq 1\right\}$. Then $B$ is a bounded closed subset of $\mathbb{R}^{2}$ and contains only the equilibrium $(1,0)$ of $(3.2 .3)$. Since $(1,0)$ is on the boundary of $B$, it follows from Lemma 2.4.2 that every positive solution of (3.2.3) converges to $(1,0)$ as $t \rightarrow \infty$. Hence, $(1,0)$ is globally asymptotically stable.

(3) Since $\mathcal{R}_{0}=1$, by Lemma 3.4.2 (3), (4.1.1) and (4.1.2), we have $|A(1,0)|=0$ and $\operatorname{tr}(A(1,0))<0$. We change the equilibrium $(1,0)$ to the origin $(0,0)$ by the change of variables $u_{1}=x-1$ and $v_{1}=y$. Note that $h=\beta-\eta$. Then system (3.2.3) becomes $\left\{\begin{array}{l}\dot{u}_{1}=\dot{x}=b-\beta\left(u_{1}+1\right) v_{1}-b\left(u_{1}+1\right)-q b v_{1}=-\beta u_{1} v_{1}-(\beta+q b) v_{1}-b u_{1}, \\ \dot{v}_{1}=\dot{y}=\beta\left(u_{1}+1\right) v_{1}-(\eta+h) v_{1}=\beta u_{1} v_{1}-(\eta+h-\beta) v_{1}=\beta u_{1} v_{1} .\end{array}\right.$ Let $\xi=(\beta+q b) b^{-1}, u_{2}=u_{1}+\xi v$ and $v_{2}=v_{1}$. Then the last system becomes

$$
\begin{aligned}
\dot{u}_{2} & =\dot{u}_{1}+\xi \dot{v}_{1}=-\beta u_{1} v_{1}-(\beta+q b) v_{1}-b u_{1}+\xi \beta u_{1} v_{1} \\
& =(\xi-1) \beta u_{1} v_{1}-(\beta+q b) v_{1}-b u_{1} \\
& =(\xi-1) \beta\left[u_{2} v_{2}-\xi v_{2}^{2}\right]-(\beta+q b) v_{2}-b\left(u_{2}-\xi v_{2}\right) \\
& =(\xi-1) \beta u_{2} v_{2}-\xi(\xi-1) \beta v_{2}^{2}-b u_{2}
\end{aligned}
$$

and $\dot{v}_{2}=\beta v_{2}\left[u_{2}-\xi v_{2}\right]=-\xi b v_{2}^{2}+\beta u_{2} v_{2}$.

Let $u=v_{2}$ and $v=u_{2}$. Then the above last two equations become

$$
\left\{\begin{array}{l}
\dot{u}=-\xi b u^{2}+\beta u v \\
\dot{v}=-b v+(\xi-1) \beta u v-\xi(\xi-1) \beta u^{2} .
\end{array}\right.
$$

Since $\varrho:=-b \neq 0$ and $a_{20}:=-\xi b \neq 0$, it follows from Lemma 2.4.3 that $(1,0)$ is a saddle-node of $(3.2 \cdot 3)$. 
Remark 4.1.1. When $\mathcal{R}_{0}<1$, Theorem 4.1.1 (2) shows that the infectionfree equilibrium $(1,0)$ is a stable node and is globally asymptotically stable. By Lemma 3.4.2 (2), we see that the biological interpretation of Theorem 4.1.1 (2) is that if $0<\beta<\eta$ with any treatment rate $h \geq 0$ or $\eta \leq \beta$ and the treatment rate $h>\beta-\eta$, then the epidemic disease will be eradicated and the epidemic can not maintain itself (see Figure $1(a)$ below).

Theorem 4.1.1 (3) is new and from its proof, we see that if we move the saddle-node $(0,0)$ back to the infection-free equilibrium $(1,0)$, then $(1,0)$ is a saddle-node in the neighbourhood of $(1,0)$, which is unstable. But we only consider the solutions in the triangle $B=\left\{(u, v) \in \mathbb{R}_{+}^{2}: u+v \leq 1\right\}$, so from the Figure $1(b)$ below, we see that all the positive solutions in $B$ converge to $(1,0)$ and $(1,0)$ is stable in $B$, which is consistent with the result when $q=h=0$ given in [5, Theorem 6.1]. Theorem 4.1.1 (1) and (2) with $h=0$ were obtained in [12, section 2].

Now, we turn our attention to the positive endemic equilibrium $(\bar{x}, \bar{y})$ given in (3.4.5) of (3.2.3). We first prove the following result which shows that under suitable conditions, $(\bar{x}, \bar{y})$ is locally asymptotically stable.

Theorem 4.1.2. If $\mathcal{R}_{0}>1$, then $(\bar{x}, \bar{y})$ is locally asymptotically stable.

Proof. Since $\mathcal{R}_{0}>1$, it follows from Theorem $3.4 .3(2)$ that $(\bar{x}, \bar{y})$ given in (3.4.5) is well defined. By (4.1.1) and (4.1.2) with $(x, y)=(\bar{x}, \bar{y})$, we have,

$$
|A(\bar{x}, \bar{y})|=\beta \bar{y}(b+r+h)+b(\eta+h)-\beta b \bar{x}=\beta \bar{y}(b+r+h) .
$$

and

$$
\operatorname{tr}(A(\bar{x}, \bar{y}))=-\beta(\bar{y}-\bar{x})-(b+h+\eta)=-(\beta \bar{y}+b)
$$



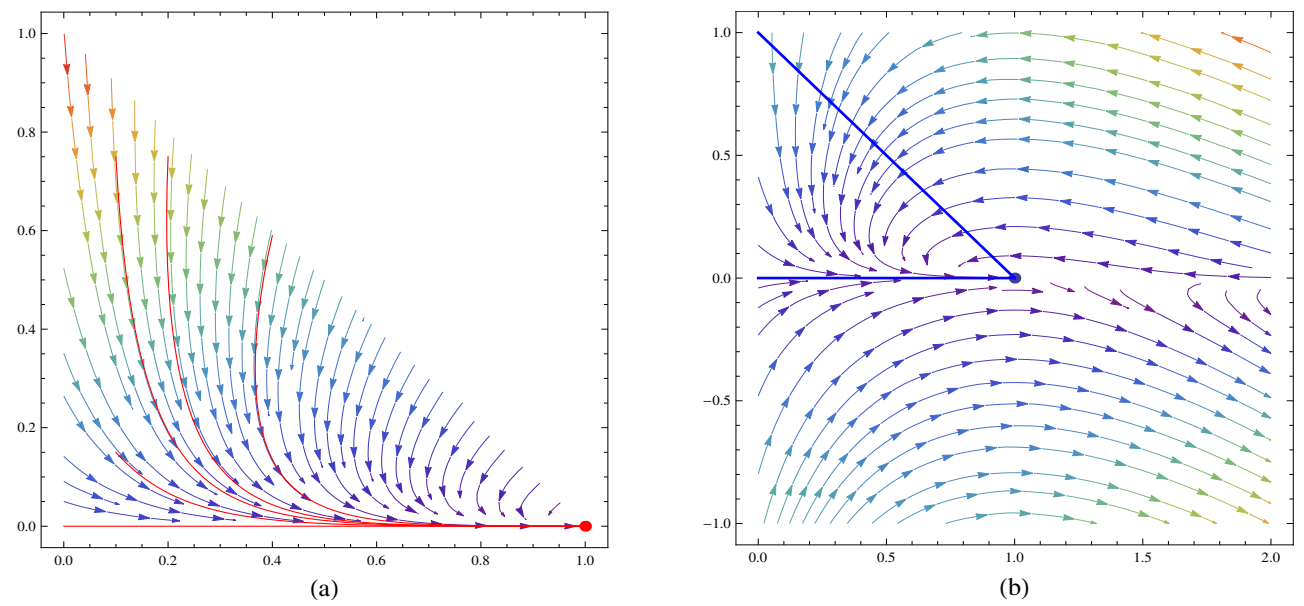

Figure 4.1: Phase portrait for 4.1.1 (2).

Since $\bar{y}>0$ and $b, r, \beta>0$, by (4.1.5) and 4.1.6, we have $|A(\bar{x}, \bar{y})|>0$ and $\operatorname{tr}(A(\bar{x}, \bar{y}))<0$. The result follows from Lemma 2.4.1 (iv).

Remark 4.1.2. By Lemma 3.4.2(1), we see that $\mathcal{R}_{0}>1$ if and only if $\eta<\beta$ and $0 \leq h<\beta-\eta$. Hence, Theorem 4.1.2 generalizes the result in [12, section 2] from $h=0$ to $h \in[0, \beta-\eta)$. The biological interpretation of Theorem 4.1.2 is that if $\eta<\beta$, then the epidemic can not be eradicated if the treatment rate $h$ is smaller than $\beta-\eta$.

We remark that it seems difficult to justify whether $(\bar{x}, \bar{y})$ is a stable node or stable focus for each $h \in[0, \beta-\eta)$. Our main goal in the rest of this paper is to find sufficient conditions on the parameters $b, r, \beta, q, h$ under which $(\bar{x}, \bar{y})$ is a stable node or stable focus. All the results obtained below are new even when $q=0$ or $h=0$.

Our first result shows that $(\bar{x}, \bar{y})$ could be a stable node or stable focus 


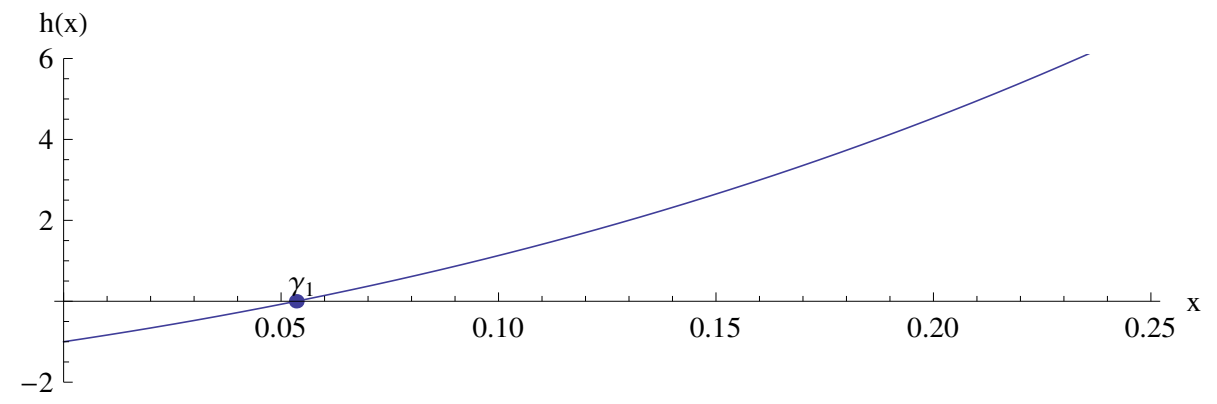

Figure 4.2: The unique solution of $h(x)=0$.

for sufficiently small $h$. To do that, we first prove the following lemmas.

Let

$$
\beta_{1}:=\frac{2(b+r)^{\frac{3}{2}}}{\sqrt{b+r}+\sqrt{r}} \quad \text { and } \beta_{2}:=\frac{2(b+r)^{\frac{3}{2}}}{\sqrt{b+r}-\sqrt{r}} .
$$

Lemma 4.1.3. Let $h(x)=16 x(1+x)^{3}-1$ for $x \in \mathbb{R}_{+}$. Then the following assertions hold.

(i) The equation $h(x)=0$ has a unique solution $\gamma_{1} \in(0.05,0.055)$.

(ii) $h(x)<0$ for $x \in\left[0, \gamma_{1}\right)$ and $h(x)>0$ for $x \in\left(\gamma_{1}, \infty\right)$.

Proof. Since $h^{\prime}(x)=16\left(1+x^{2}\right)(1+4 x)>0$ for $x \in[0, \infty), h$ is strictly increasing on $[0, \infty)$. Since $h(0.05)<0$ and $h(0.055)>0$, the result $(i)$ and (ii) follow.

The graph of the function $h$ in Lemma 4.1 .3 is given in Figure 2, and we can see that the unique solution of $h(x)=0$ lies between 0.05 and 0.055 .

Lemma 4.1.4. (1) If $b>0, r>0$, then

$$
r<\beta_{1}-b<b+r<\beta_{2}-b
$$


and the following assertions hold.

(i) If $r<\beta<\beta_{1}-b$, then $0<q_{1}<1<\frac{\beta_{1}-\beta}{b}<\frac{\beta_{2}-\beta}{b}$.

(ii) If $\beta_{1}-b<\beta \leq b+r$, then $0<q_{1}<\frac{\beta_{1}-\beta}{b}<1$ and $\frac{\beta_{2}-\beta}{b}>1$.

(2) If $b>0$ and $r>\gamma_{1} b$, then

$$
r<\beta_{1}-b<b+r<\beta_{1}<\beta_{2}-b<\beta_{2}
$$

and the following assertions hold.

(i) If $b+r<\beta \leq \beta_{1}$, then $0 \leq \frac{\beta_{1}-\beta}{b}<1$ and $\frac{\beta_{2}-\beta}{b}>1$.

(ii) If $\beta_{1}<\beta<\beta_{2}-b$, then $\frac{\beta_{1}-\beta}{b}<0$ and $\frac{\beta_{2}-\beta}{b}>1$.

(iii) If $\beta_{2}-b<\beta<\beta_{2}$, then $\frac{\beta_{1}-\beta}{b}<0$ and $0<\frac{\beta_{2}-\beta}{b}<1$.

(3) If $0<r<\gamma_{1} b$, then

$$
r<\beta_{1}-b<b+r<\beta_{2}-b<\beta_{1}<\beta_{2}
$$

and the following assertions hold.

(i) If $b+r<\beta<\beta_{2}-b$, then $0<\frac{\beta_{1}-\beta}{b}<1$ and $\frac{\beta_{2}-\beta}{b}>1$.

(ii) If $\beta_{2}-b<\beta \leq \beta_{1}$, then $0 \leq \frac{\beta_{1}-\beta}{b}<1$ and $0<\frac{\beta_{2}-\beta}{b}<1$.

(iii) If $\beta_{1}<\beta<\beta_{2}$, then $\frac{\beta_{1}-\beta}{b}<0$ and $0<\frac{\beta_{2}-\beta}{b}<1$.

(4) If $r=\gamma_{1} b$, then

$$
r<\beta_{1}-b<b+r<\beta_{2}-b=\beta_{1}<\beta_{2}
$$

and the following assertions hold.

(i) If $b+r<\beta<\beta_{2}-b$, then $0<\frac{\beta_{1}-\beta}{b}<1$ and $\frac{\beta_{2}-\beta}{b}>1$.

(ii) If $\beta_{1}<\beta<\beta_{2}$, then $\frac{\beta_{1}-\beta}{b}<0$ and $0<\frac{\beta_{2}-\beta}{b}<1$. 
Proof. (1) Let $b>0$ and $r>0$. Since

$$
\beta_{1}=\frac{2(b+r)^{\frac{3}{2}}}{\sqrt{b+r}+\sqrt{r}}=\frac{2(b+r)}{1+\sqrt{\frac{r}{b+r}}}>\frac{2(b+r)}{1+1}=b+r,
$$

it follows that $r<\beta_{1}-b$. Since

$$
\begin{aligned}
\beta_{1}-(2 b+r) & =\frac{2(b+r)}{1+\sqrt{\frac{r}{b+r}}}-(2 b+r)=\frac{2(b+r)-(2 b+r)\left[1+\sqrt{\frac{r}{b+r}}\right]}{1+\sqrt{\frac{r}{b+r}}} \\
& =\frac{r-(2 b+r) \sqrt{\frac{r}{b+r}}}{1+\sqrt{\frac{r}{b+r}}}=\frac{r^{2}-(2 b+r)^{2} \frac{r}{b+r}}{\left[1+\sqrt{\frac{r}{b+r}}\right]\left[r+(2 b+r) \sqrt{\frac{r}{b+r}}\right]} \\
& =\frac{r^{2}(b+r)-r(2 b+r)^{2}}{(b+r)\left[1+\sqrt{\frac{r}{b+r}}\right]\left[r+(2 b+r) \sqrt{\frac{r}{b+r}}\right]} \\
& =-\frac{r b(4 b+3 r)}{(b+r)\left[1+\sqrt{\frac{r}{b+r}}\right]\left[r+(2 b+r) \sqrt{\frac{r}{b+r}}\right]}<0 .
\end{aligned}
$$

we have $\beta_{1}-b<b+r$. Since

$$
\begin{aligned}
\beta_{2}-(2 b+r) & =\frac{2(b+r)}{1-\sqrt{\frac{r}{b+r}}}-(2 b+r)=\frac{2(b+r)-(2 b+r)\left[1-\sqrt{\frac{r}{b+r}}\right]}{1-\sqrt{\frac{r}{b+r}}} \\
& =\frac{r+(2 b+r) \sqrt{\frac{r}{b+r}}}{1-\sqrt{\frac{r}{b+r}}}>0,
\end{aligned}
$$

we have $b+r<\beta_{2}-b$. Hence, (4.1.7) holds. Note that $q_{1}=(b+r-\beta) b^{-1}$. By (4.1.7), it is steadily verified that the results $(i)$ - $(i i)$ hold.

Let $b>0$ and $r>0$. By definition of $\beta_{1}$ and $\beta_{2}$ we have

$$
\begin{aligned}
\beta_{2}-b-\beta_{1} & =\frac{2(b+r)^{\frac{3}{2}}}{\sqrt{b+r}-\sqrt{r}}-\frac{2(b+r)^{\frac{3}{2}}}{\sqrt{b+r}+\sqrt{r}}-b=\frac{4 \sqrt{r}(b+r)^{\frac{3}{2}}}{b}-b \\
& =\frac{4 \sqrt{r}(b+r)^{\frac{3}{2}}-b^{2}}{b}=\frac{16 r(b+r)^{3}-b^{4}}{b\left[4 \sqrt{r}(b+r)^{\frac{3}{2}}+b^{2}\right]}=\frac{b^{3}\left[16 \frac{r}{b}\left(1+\frac{r}{b}\right)^{3}-1\right]}{4 \sqrt{r}(b+r)^{\frac{3}{2}}+b^{2}} \\
& =\frac{b^{3} h\left(\frac{r}{b}\right)}{4 \sqrt{r}(b+r)^{\frac{3}{2}}+b^{2}}
\end{aligned}
$$

(2) If $b>0$ and $r>\gamma_{1} b$, then $\frac{r}{b}>\gamma_{1}$ and by Lemma $4.1 .3, h\left(\frac{r}{b}\right)>0$. It follows from (4.1.11) that $\beta_{1}<\beta_{2}-b$. Since $\beta_{1}>b+r$, we have $r<\beta_{1}-b$. 
Hence, (4.1.8) holds. By (4.1.8), it is steadily verified that the results $(i)$-(iii) hold.

(3) If $0<r<\gamma_{1} b$, then $\frac{r}{b}<\gamma_{1}$ and by Lemma 4.1.3, $h\left(\frac{r}{b}\right)<0$. It follows from (4.1.11) that $\beta_{2}-b<\beta_{1}$. It is obvious that $\beta_{1}<\beta_{2}$. Since

$$
\beta_{2}=\frac{2(b+r)^{\frac{3}{2}}}{\sqrt{b+r}-\sqrt{r}}=\frac{2(b+r)}{1-\sqrt{\frac{r}{b+r}}}>\frac{2(b+r)}{1-0}>2 b+r,
$$

we obtain $b+r<\beta_{2}-b$. It has been proved in (1) that $\beta_{1}-b<b+r$. Hence, (4.1.9) holds. By (4.1.9), it is steadily verified that the results $(i)$-(iii) hold.

(4) If $r=\gamma_{1} b$, then $\beta_{1}=\beta_{2}-b$. Hence, (4.1.10) holds and (i) and (ii) hold.

Let $(\bar{x}, \bar{y})=\left(\frac{\eta+h}{\beta}, \frac{b(\beta-\eta-h)}{\beta(b+r+h)}\right)$ be same as in (3.4.5) and let

$$
\Delta(q, h)=\operatorname{tr}(A(\bar{x}, \bar{y}))^{2}-4|A(\bar{x}, \bar{y})|
$$

The following result gives a useful formula which will be used later.

Lemma 4.1.5. If $\mathcal{R}_{0}>1$, then

$$
\Delta(q, h)=\frac{b}{(b+r+h)^{2}}\left[b(\beta+q b)^{2}-4(b+r+h)^{2}(\beta+q b)+4(b+r+h)^{3}\right] .
$$

Proof. Noting that $\eta=b+r-q b$, we have

$$
\beta \bar{y}+b=\frac{b(\beta+q b)}{b+r+h} \quad \text { and } 4 \beta \bar{y}(b+r+h)=(\beta+q b)-(b+r+h) .
$$


This, together with (4.1.5) and (4.1.6), implies

$$
\begin{aligned}
\Delta(q, h) & :=\operatorname{tr}(A(\bar{x}, \bar{y}))^{2}-4|A(\bar{x}, \bar{y})|=(\beta \bar{y}+b)^{2}-4 \beta \bar{y}(b+r+h) \\
& =\frac{b^{2}(\beta+q b)^{2}}{(b+r+h)^{2}}-4 b[(\beta+q b)-(b+r+h)] \\
& =\frac{b}{(b+r+h)^{2}}\left[b(\beta+q b)^{2}-4(b+r+h)^{2}(\beta+q b)+4(b+r+h)^{3}\right] .
\end{aligned}
$$

The result follows.

Theorem 4.1.6. (1) Assume that one of the following conditions $\left(H_{1}\right)-\left(H_{4}\right)$ holds.

$\left(H_{1}\right) b>0, r>0, \beta_{1}-b<\beta \leq b+r$ and $\frac{\beta_{1}-\beta}{b}<q \leq 1$.

$\left(H_{2}\right) b>0, r>\gamma_{1} b$ and one of the following conditions holds.

(i) $b+r<\beta \leq \beta_{1}$ and $\frac{\beta_{1}-\beta}{b}<q \leq 1$.

(ii) $\beta_{1}<\beta<\beta_{2}-b$ and $0 \leq q \leq 1$.

(iii) $\beta=\beta_{2}-b$ and $0 \leq q<1$.

(iv) $\beta_{2}-b<\beta<\beta_{2}$ and $0 \leq q<\frac{\beta_{2}-\beta}{b}$.

$\left(H_{3}\right) \quad b>0,0<r<\gamma_{1} b$ and one of the following conditions holds.

(i) $b+r<\beta<\beta_{2}-b$ and $\frac{\beta_{1}-\beta}{b}<q \leq 1$.

(ii) $\beta=\beta_{2}-b$ and $\frac{\beta_{1}-\beta}{b}<q<1$.

(iii) $\beta_{2}-b<\beta \leq \beta_{1}$ and $\frac{\beta_{1}-\beta}{b}<q<\frac{\beta_{2}-\beta}{b}$.

(iv) $\beta_{1}<\beta<\beta_{2}$ and $0 \leq q<\frac{\beta_{2}-\beta}{b}$. 
$\left(H_{4}\right) b>0, r=\gamma_{1} b$ and one of the following conditions holds.

(i) $b+r<\beta<\beta_{2}-b$ and $\frac{\beta_{1}-\beta}{b}<q \leq 1$.

(ii) $\beta=\beta_{2}-b$ and $\frac{\beta_{1}-\beta}{b}<q<1$.

(iii) $\beta_{2}-b<\beta<\beta_{2}$ and $0 \leq q<\frac{\beta_{2}-\beta}{b}$.

Then there exists $h_{0} \in(0, \beta-\eta)$ such that $(\bar{x}, \bar{y})$ is a stable focus of $(3.2 .3)$ for $h \in\left[0, h_{0}\right)$.

(2) Assume that $b>0, r>0$ and one of the following conditions holds.

(i) $r<\beta<\beta_{1}-b$ and $q_{1}<q \leq 1$.

(ii) $\beta=\beta_{1}-b$ and $q_{1}<q<1$.

(iii) $\beta_{2}<\beta<\infty$ and $0 \leq q \leq 1$

(iv) $\beta_{2}=\beta$ and $0<q \leq 1$.

Then there exists $h_{1} \in(0, \beta-\eta)$ such that $(\bar{x}, \bar{y})$ is a stable node of $(3.2 .3)$ for $h \in\left[0, h_{1}\right)$.

Proof. Under the conditions of $\left(H_{1}\right)$, by ( $\left.i i\right)$ of Lemma 4.1.4 (1), $r<\beta \leq b+r$ and $q_{1}<q \leq 1$. This, together with Lemma 3.4.1 (2), implies $\eta<\beta$. Similarly, by Lemma $4.1 .4(2),(3),(4)$, each of the hypotheses in $\left(H_{2}\right)-\left(H_{4}\right)$ implies $b+r<\beta$ and $0 \leq q \leq 1$. By Lemma 3.4.1 (2), we obtain $\eta<\beta$.

Let $0 \leq h<\beta-\eta$. By (4.1.13), we have

$$
\Delta(q, h)=\frac{b^{2} \Gamma(h)}{(b+r+h)^{2}},
$$

where

$$
\Gamma(q, h)=(\beta+q b)^{2}-\frac{4(b+r+h)^{2}(\beta+q b)}{b}+\frac{4(b+r+h)^{3}}{b} .
$$


We prove

$$
\Gamma(q, 0)=b^{2}\left(q-\frac{\beta_{1}-\beta}{b}\right)\left(q-\frac{\beta_{2}-\beta}{b}\right) .
$$

Indeed, by (4.1.15) we have

$$
\begin{aligned}
\Gamma(q, 0) & =(\beta+q b)^{2}-\frac{4(b+r)^{2}(\beta+q b)}{b}+\frac{4(b+r)^{3}}{b} \\
& =\left[\beta+q b-\frac{2(b+r)^{2}}{b}\right]^{2}-\frac{4 r(b+r)^{3}}{b^{2}} \\
& =\left[\beta+q b-\frac{2(b+r)^{2}}{b}+\frac{2 \sqrt{r}(b+r)^{\frac{3}{2}}}{b}\right]\left[\beta+q b-\frac{2(b+r)^{2}}{b}-\frac{2 \sqrt{r}(b+r)^{\frac{3}{2}}}{b}\right] \\
& =\left[\beta+q b-\frac{2(\sqrt{b+r}-\sqrt{r})(b+r)^{\frac{3}{2}}}{b}\right]\left[\beta+q b-\frac{2(\sqrt{b+r}+\sqrt{r})(b+r)^{\frac{3}{2}}}{b}\right] \\
& =\left[\beta+q b-\frac{2 b(b+r)^{\frac{3}{2}}}{b(\sqrt{b+r}+\sqrt{r})}\right]\left[\beta+q b-\frac{2 b(b+r)^{\frac{3}{2}}}{b(\sqrt{b+r}-\sqrt{r})}\right] \\
& =\left(\beta+q b-\beta_{1}\right)\left(\beta+q b-\beta_{2}\right)
\end{aligned}
$$

and (4.1.16) holds.

We prove that under each of the conditions in $\left(H_{1}\right)-\left(H_{4}\right)$,

$$
\frac{\beta_{1}-\beta}{b}<q<\frac{\beta_{2}-\beta}{b}
$$

$\left(H_{1}\right)$ If $b>0, r>0, \beta_{1}-b<\beta<b+r$ and $\frac{\beta_{1}-\beta}{b}<q \leq 1$. then by $(i i)$ of Lemma 4.1.4 (1), we have

$$
\frac{\beta_{1}-\beta}{b}<q \leq 1<\frac{\beta_{2}-\beta}{b}
$$

$\left(H_{2}\right)(i)$ If $b+r<\beta<\beta_{1}$ and $\frac{\beta_{1}-\beta}{b} \leq q \leq 1$, then by $(i)$ of Lemma 4.1 .4 $(2)$

$$
\frac{\beta_{1}-\beta}{b}<q \leq 1<\frac{\beta_{2}-\beta}{b}
$$


(ii) If $\beta_{1}<\beta<\beta_{2}-b$ and $0 \leq q \leq 1$, then by (ii) of Lemma 4.1.4 (2),

$$
\frac{\beta_{1}-\beta}{b}<0 \leq q \leq 1<\frac{\beta_{2}-\beta}{b}
$$

(iii) If $\beta_{2}-b<\beta<\beta_{2}$ and $0 \leq q<\frac{\beta_{2}-\beta}{b}$, then by (iii) of Lemma 4.1.4 $(2)$,

$$
\frac{\beta_{1}-\beta}{b}<0 \leq q<\frac{\beta_{2}-\beta}{b}
$$

Hence, under each of the conditions $(i),(i i)$ and $(i i i)$ in $\left(H_{2}\right),(4.1 .17)$ holds. Similarly, (4.1.17) holds under each of the conditions in $\left(H_{3}\right)$ or $\left(H_{4}\right)$. By (4.1.16) and (4.1.17), we see that $\Gamma(q, 0)<0$. It follows from the continuity of $\Gamma$ that there exists $h_{0} \in(0, \beta-\eta)$ such that $\Gamma(h)<0$ for $h \in\left[0, h_{0}\right)$, and by (4.1.14), $\Delta(q, h)<0$ for $h \in\left[0, h_{0}\right)$. By Theorem 4.1.2 (1), (4.1.5) and (4.1.6) we see that for $h \in\left[0, h_{0}\right),|A(\bar{x}, \bar{y})|>0$ and $\operatorname{tr}(\bar{x}, \bar{y})<0$. The result follows from Lemma 2.4.1 ( $\mathrm{iii}$ ).

(2) (i) If $r<\beta<\beta_{1}-b$ and $q_{1}<q \leq 1$, then by ( $i$ ) of Lemma 4.1.4 (1), we have

$$
1<\frac{\beta_{1}-\beta}{b}<\frac{\beta_{2}-\beta}{b}
$$

(ii) If $\beta=\beta_{1}-b$ and $q_{1}<q<1$, then

$$
q_{1}<q<1=\frac{\beta_{1}-\beta}{b}<\frac{\beta_{2}-\beta}{b} .
$$

(iii) If $\beta_{2}<\beta<\infty$ and $0 \leq q \leq 1$, then we have

$$
\frac{\beta_{1}-\beta}{b}<\frac{\beta_{2}-\beta}{b}<0
$$

By (4.1.16) and each of (4.1.18), (4.1.19) and (4.1.20), we have $\Gamma(0)>0$. It follows from the continuity of $\Gamma$ that there exists $h_{1} \in(0, \beta-\eta)$ such that 

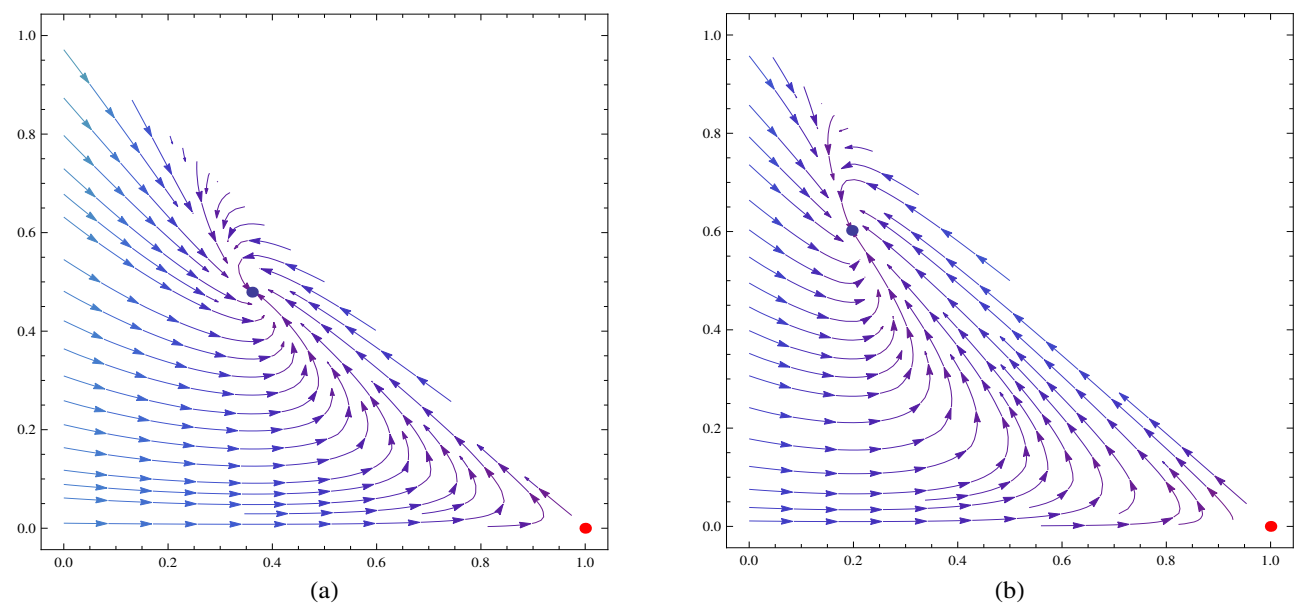

Figure 4.3: Phase portrait of theorem 4.1.6 $\left(\mathrm{H}_{2}\right)(\mathrm{ii})$ and $2(\mathrm{iii})$ respectively.

$\Gamma(h)>0$ for $h \in\left[0, h_{1}\right)$. It follows from (4.1.14) that $\Delta(h)>0$ for $h \in\left[0, h_{1}\right)$. The result follows from Lemma 2.4.1 $(i i)$.

The simulation results for Theorem 4.1.6 $\left(\mathrm{H}_{2}\right)(\mathrm{ii})$ and $(2)(\mathrm{iii})$ are given in the following figure 3 .

By Theorem 4.1.6 with $h=q=0$, we see that if $\beta_{1}<\beta<\beta_{2}$, then $(\bar{x}, \bar{y})$ is a stable focus of (3.2.3) with $h=q=0$ and if $\beta>\beta_{2}$, then $(\bar{x}, \bar{y})$ is a stable node of (3.2.3) with $h=q=0$. Theorem 4.1 .6 with $h=q=0$ is inconclusive if $b+r<\beta \leq \beta_{1}$ or $\beta=\beta_{2}$.

Using the formulas (4.1.14) and (4.1.16) obtained above, we can provide a direct proof to the following new result on the classic model (3.2.3) with $h=q=0$.

Theorem 4.1.7. (1) If $\beta_{1}<\beta<\beta_{2}$, then $(\bar{x}, \bar{y})$ is a stable focus of (3.2.3) with $h=q=0$. 
(2) If either $b+r<\beta \leq \beta_{1}$ or $\beta \geq \beta_{2}$, then $(\bar{x}, \bar{y})$ is a stable node of (3.2.3) with $h=q=0$.

Proof. By (4.1.14) and (4.1.16), we have

$$
\Delta(0,0)=\frac{b^{2}}{(b+r)^{2}}\left(\beta-\beta_{1}\right)\left(\beta-\beta_{2}\right) .
$$

(1) If $\beta_{1}<\beta<\beta_{2}$, then $\Delta(0,0)<0$. By Lemma 2.4.1 (iii), the result (1) holds.

(2) If either $b+r<\beta \leq \beta_{1}$ or $\beta \geq \beta_{2}$, then $\Delta(0,0) \geq 0$ and the result (2) follows from Lemma 2.4.1 (ii).

Theorem 4.1.6 shows that the interior equilibrium $(\bar{x}, \bar{y})$ of $(3.2 .3)$ can be a stable focus or a stable node for sufficiently small $h$. However, Theorem 4.1.6 does not provide any upper bounds for $h$. Hence, the question is that under which range of $h$, can the interior equilibrium $(\bar{x}, \bar{y})$ be a stable focus or a stable node?

In the following, we enhance the result (iii) of Theorem 4.1.6 (2) and partially answer the above question. We provide a range of $h$ under which $(\bar{x}, \bar{y})$ is a stable node of (3.2.3). To do this, we first prove the following lemma.

Let

$$
\beta_{0}=\frac{4(b+r)^{2}}{b} \text { and } h_{1}=\frac{\sqrt{b(\beta+q b)}}{2}-b-r .
$$

Lemma 4.1.8. (1) $\beta_{2}<\beta_{0}-b$.

(2) If $\beta_{0}-b \leq \beta \leq \infty$ and $\max \left\{0, \frac{\beta_{0}-\beta}{b}\right\} \leq q \leq 1$, then $0 \leq h_{1}<\beta-\eta$. 
Proof. (1) Since

$$
\begin{gathered}
\frac{2(b+r)}{b}=\frac{1}{1+\sqrt{\frac{r}{b+r}}}+\frac{1}{1-\sqrt{\frac{r}{b+r}}}, \\
\beta_{0}-\beta_{2}-b=\frac{4(b+r)^{2}}{b}-\frac{2(b+r)}{1-\sqrt{\frac{r}{b+r}}}-b=\frac{2(b+r)}{1+\sqrt{\frac{r}{b+r}}}-b \\
=\frac{2(b+r)-b\left(1+\sqrt{\frac{r}{b+r}}\right)}{1+\sqrt{\frac{r}{b+r}}}=\frac{b\left(1-\sqrt{\frac{r}{b+r}}\right)+2 r}{1+\sqrt{\frac{r}{b+r}}}>0 .
\end{gathered}
$$

(2) We first prove that under the given hypotheses,

$$
b-4 \beta-4 q b<0 \text {. }
$$

In fact, if $\frac{\beta_{0}-\beta}{b} \geq 0$, then $q \geq \frac{\beta_{0}-\beta}{b}$ and

$$
b-4 \beta-4 q b \leq b-4 \beta-4\left(\beta_{0}-\beta\right)=b-4 \beta_{0}=\frac{b^{2}-4(b+r)^{2}}{b}<0 .
$$

If $\frac{\beta_{0}-\beta}{b}<0$, then $q \geq 0$ and

$$
b-4 \beta-4 q b \leq b-4 \beta \leq b-4\left(\beta_{0}-b\right)=\frac{5 b^{2}-16(b+r)^{2}}{b}<-11 b<0 .
$$

Next, we prove that $h_{1}<\beta-\eta$. Indeed, since

$$
\begin{aligned}
h_{1}-(\beta-\eta) & =\frac{\sqrt{b(\beta+q b)}-2(b+r)}{2}-(\beta-b-r+q b) \\
& =\frac{\sqrt{b(\beta+q b)}}{2}-(\beta+q b)=\frac{(\beta+q b)[b-4 \beta-4 q b)]}{2[\sqrt{b(\beta+q b)}+4(\beta+q b)]} .
\end{aligned}
$$

This, together with (4.1.21), implies $h_{1}<\beta-\eta$. Finally, we prove that 
$h_{1} \geq 0$. In fact, since

$$
\begin{aligned}
h_{1} & =\frac{\sqrt{b(\beta+q b)}-2(b+r)}{2}=\frac{b(\beta+q b)-4(b+r)^{2}}{2[\sqrt{b(\beta+q b)}+2(b+r)]} \\
& =\frac{b(\beta+q b)-4(b+r)^{2}}{2[\sqrt{b(\beta+q b)}+2(b+r)]}=\frac{b\left[(\beta+q b)-\beta_{0}\right]}{2[\sqrt{b(\beta+q b)}+2(b+r)]} \\
& =\frac{b^{2}\left(q-\frac{\beta_{0}-\beta}{b}\right)}{2[\sqrt{b(\beta+q b)}+2(b+r)]},
\end{aligned}
$$

it follows from $q \geq \max \left\{0, \frac{\beta_{0}-\beta}{b}\right\}$ that $q-\frac{\beta_{0}-\beta}{b} \geq 0$ and $h_{1} \geq 0$.

Theorem 4.1.9. If $\beta_{0}-b \leq \beta \leq \infty, \max \left\{0, \frac{\beta_{0}-\beta}{b}\right\} \leq q \leq 1$ and $0 \leq h \leq h_{1}$, then $(\bar{x}, \bar{y})$ is a stable node of $(3.2 .3)$.

Proof. By Lemma 4.1.4, we see that $b+r<\beta_{2}$ and by Lemma 4.1.8, $b+r<$ $\beta_{2}<\beta_{0}-b \leq \beta$. Hence, by Lemma 3.4.1 (ii), Lemma 3.4.2 and Theorem 3.4.3 (2) we see that for $\max \left\{0, \frac{\beta_{0}-\beta}{b}\right\} \leq q \leq 1,(\bar{x}, \bar{y})$ given in (3.4.5) is well defined. It is easy to verify that if $h \leq h_{1}$, then $2(b+r+h) \leq \sqrt{b(\beta+q b)}$ and

$$
b(\beta+q b)-4(b+r+h)^{2} \geq 0 .
$$

This, together with (4.1.13), implies

$\Delta(q, h)=\frac{b}{(b+r+h)^{2}}\left\{(\beta+q b)\left[b(\beta+q b)-4(b+r+h)^{2}\right]+4(b+r+h)^{3}\right\}>0$.

The result follows from Lemma 2.4.1 (ii).

Theorem 4.1.9 provides a range for $h$ under which $(\bar{x}, \bar{y})$ is a stable node of (3.2.3), see Figure 4 below for a simulation result. By Lemma 4.1.8 (1), we see that under the hypothesis of Theorem 4.1.9: $\beta_{0}-b \leq \beta \leq \infty$, we 


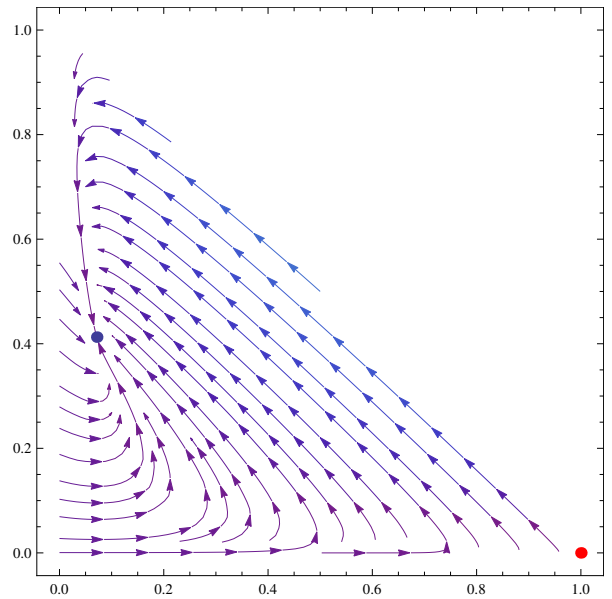

Figure 4.4: Phase portrait of theorem 4.1.9.

have $\beta_{2}<\beta_{0}-b \leq \beta$. Hence, Theorem 4.1.9 strengthens the result (iii) of Theorem 4.1.6 (2) which holds for sufficiently small $h$. 


\section{Chapter 5}

\section{Discussion}

We studied an SIR epidemic model with horizontal, vertical transmission and a linear treatment rate. We proved that the basic reproduction number $\mathcal{R}_{0} \leq 1$, the model has a unique disease-free equilibrium $(1,0)$, and when $\mathcal{R}_{0}>1$, the model has both a disease-free equilibrium $(1,0)$ and an interior (epidemic) equilibrium $(\bar{x}, \bar{y})$. Secondly, we studied the phase portraits near the equilibria. Our results on the infection-free equilibrium $(1,0)$ provide more detailed understandings on its dynamical properties. For example, we showed that when $\mathcal{R}_{0}>1,(1,0)$ is a saddle, when $\mathcal{R}_{0}<1,(1,0)$ is a stable node, and when $\mathcal{R}_{0}=1$, it is a saddle-node and is stable in the triangle region $\left\{(u, v) \in \mathbb{R}_{+}: u+v \leq 1\right\}$. While the previous results in [12] only focused on its local stability.

For the epidemic equilibrium $(\bar{x}, \bar{y})$, we provide sufficient conditions on the parameters and prove that the epidemic equilibria is a stable focus or a stable node under different conditions. 


\section{Bibliography}

[1] A. A. Andronov, E. A. Leontovich, I. I. Gordon and A. G. Maier, Qualitative Theory of Second-Order Dynamical Systems, John Wiley and Sons, New York, 1973.

[2] K. Bellenir and P. Dresser, Contagious and Non-Contagious Infectious Diseases Sourcebook, Health Science Series 8, Omnigraphics Inc., Detroit, 1996.

[3] S. Busenberg and K. Cooke, Vertically transmitted diseases. Models and Dynamics, Biomathematics 23, Springer-Verlag, New York, 1993.

[4] C. M. Heggerud and K. Q. Lan, Local stability analysis of ratiodependent predator-prey models with predator harvesting rates, Appl. Math. Comput., 270(2015), 349-357.

[5] H. W. Hethcote, Three basic epidemiological models, Applied mathematical ecology (Trieste, 1986), 119-144, Biomathematics, 18, Springer, Berlin, 1989. 
[6] S. Jana, S. K. Nandi and T. K. Kar, Complex dynamics of an SIR epidemic model with saturated incidence rate and treatment, Acta Biotheor. 64 (2016), 65-84.

[7] H. Laarabi, A. Abta and H. Hattaf, Optimal control of a delayed SIRS epidemic model with vaccination and treatment, Acta Biotheor. 63 (2015), 87-97.

[8] K. Q. Lan and C. R. Zhu, Phase portraits, Hopf bifurcations and limit cycles of the Holling-Tanner models for predator-prey interactions, Nonlinear Analysis RWA, 12(2011), 1961-1973.

[9] M. Y. Li, H. L. Smith and L. Wang, Global dynamics of an SEIR epidemic with vertical transmission, SIAM J. Appl. Math., 62(2001), 58-69.

[10] G. P. Luo, K. Q. Lan and C. R. Zhu, Bifurcations of ratio-dependent predator-prey Holling type III systems with harvesting rates, J. Nonlinear Funct. Anal. 2014 (2014), Article ID 16.

[11] G. P. Luo, C. R. Zhu and K. Q. Lan, Dynamics of SIR epidemic models with horizontal and vertical transmissions and constant treatment rates, J. Appl. Anal. Comput., in press.

[12] X. Meng and L. Chen, The dynamics of a new SIR epidemic model concerning pulse vaccination strategy, Appl. Math. Comput., 197(2008), 582-597. 
[13] L. Perko, Differential Equations and Dynamical Systems, SpringerVerlag, New York, 1996.

[14] E. Rivero-Esquivel, E. Avila-Vales, and G. Garca-Almeida, Stability and bifurcation analysis of a SIR model with saturated incidence rate and saturated treatment, Math. Comput. Simulation. 121 (2016), 109-132.

[15] H. L. Smith and P. Waltman, The Theory of the Chemostat, Cambridge University Press, 1995.

[16] G. Teschl, Ordinary Differential Equations and Dynamical Systems, American Mathematical Society, 2012.

[17] W. Wang and S. Ruan, Bifurcations in an epidemic model with constant removal rate of the infectives, J. Math. Anal. Appl., 291(2004), 775-793.

[18] X. Zhang and X. Liu, Backward bifurcation of an epidemic model with saturated treatment function, J. Math. Anal. Appl. 348 (2008), 433-443.

[19] C. R. Zhu and K. Q. Lan, Phase portraits, Hopf bifurcations and limit cycles of Leslie-Gower predator-prey systems with harvesting rates, Discrete Contin. Dyn. Syst. Ser. B, 14(2010), 289-306.

[20] Allen, L. J. S. (2007). An introduction to mathematical biology. Upper Saddle River, NJ: Pearson/Prentice Hall,

[21] Thi Doan Doan, Changrong Zhu and Kunquan Lan, Phase plane analysis of the Susceptible-Infected- Removed-Susceptible (SIRS) epidemic 
models with nonlinear incidence rates, Journal of Nonlinear Functional Analysis 2016 (2016), Article ID 30. 